\title{
The Use of Magnetic Resonance Imaging-guided Focused Ultrasound in Movement Disorders- A Review
}

\author{
Lauren S Talman and Binit B Shah
}

Department of Neurology, University of Virginia, Charlottesville, VA, US

DOl: https://doi.org/10.17925/USN.2017.13.02.74

\section{Keywords}

Essential tremor, Parkinson's disease, tremor, thalamotomy, focused ultrasound

Disclosure: Lauren S Talman and Binit B Shah have nothing to declare in relation to this article. No funding was received in the publication of this article.

Authorship: All named authors meet the International Committee of Medical Journal Editors (ICMJE) criteria for authorship of this manuscript, take responsibility for the integrity of the work as a whole, and have given final approval for the version to be published. open Access: This article is published under the Creative Commons Attribution Noncommercial License, which permits any noncommercial use, distribution, adaptation, and reproduction provided the original author(s) and source are given appropriate credit.

Received: May 19, 2017

Accepted: July 6, 2017

Citation: US Neurology, 2017;13(2):74-7

Corresponding Author: Binit B Shah, MD. PO Box 800394 , McKim Hall 2nd Floor, Charlottesville, VA 22908, US.

E: binitshah@virginia.edu
Over the past few decades, the use of surgical techniques for the treatment of movement disorders has become well established. Ablative lesioning procedures, including radiofrequency ablation and gamma knife radiosurgery, were utilized in practice starting in the 1960s, and deep brain stimulation (DBS) was introduced in the 1980s. Indications for these procedures have included motor fluctuations and levodopa-induced dyskinesia in Parkinson's disease (PD), medication-refractory essential tremor (ET) or, more rarely, dystonia. Here, we review the emerging technology of magnetic resonance imaging (MRI)-guided focused ultrasound (MRgFUS) with regard to its background, current evidence for use, and potential future applications.

\section{Introduction of technology}

FUS is emerging as a newly recognized surgical option in the field of movement disorders. Though robust trials studying the clinical application of MRgFUS have only recently been carried out, its roots date back to the 1950s when the Fry brothers of Illinois described use of ultrasound energy for precise lesioning of the internal capsule of cats. ${ }^{1}$ While this technique initially required craniectomy for accurate energy focusing, the advent of a phased array transducer has allowed for an incisionless procedure with retained precision.

The technique for use of MRgFUS has become largely standardized. A patient's head is shaved to avoid possible heat trapping between hair and scalp and a water-filled elastic cap is placed over the scalp. Lesions are targeted using 1,024 ultrasonic transducers arranged in a hemispheric orientation (Figure 1). The utility of MRgFUS as a lesioning technique is based on studies showing that tissue changes occur when temperatures reach $55-65^{\circ} \mathrm{C} .^{2}$ In practice, initial energy pulses are applied to reach a non-ablative temperature of $40-45^{\circ} \mathrm{C}$. Lesion accuracy is determined by clinical examination of the patient while temperature monitoring is provided through MRI thermometry. A series of sonications are then delivered to reach goal temperature and clinical effect. Prior studies by Jeanmonod et al. have demonstrated accuracy of this technique to within $1 \mathrm{~mm}$ of a target lesion. ${ }^{3}$ This precision, along with the ability to monitor treatment effect in real-time, have made MRgFUS an attractive tool for treatment of multiple movement disorders with the most robust data emerging from trials in ET.

\section{Essential tremor}

ET is one of the most common movement disorders worldwide estimated to affect almost $5 \%$ of individuals over 65 years old. ${ }^{4}$ The tremor is fast frequency $(4-12 \mathrm{~Hz})$ with postural and kinetic components; it primarily affects the arms but can involve the head, voice, trunk, and legs. ${ }^{5}$ While the diagnosis does not shorten a person's lifespan, the tremor can impair function, lead to disability 
and diminish overall quality of life. Pharmacologic therapy includes first-line agents propranolol and primidone, and second-line agents including topiramate, gabapentin, atenolol, alprazolam, and others. ${ }^{6}$ While medication is effective for most patients with ET, large population studies have suggested that $25-50 \%$ of ET is refractory to medication. ${ }^{5}$

Stimulation and ablation of the thalamic ventral intermediate nucleus (Vim) has been shown to effectively reduce tremor in ET.? Multiple trials report clinical improvement of ET following radiofrequency thalamotomy, ${ }^{8,9}$ gamma knife radiosurgery ${ }^{10-13}$ and DBS. ${ }^{14}$ Comparison studies of these varying techniques are limited. Schuurman et al.'s randomized study comparing unilateral radiofrequency thalamotomy versus bilateral DBS in patients with $\mathrm{PD}$, ET, or multiple sclerosis suggests that thalamic stimulation is more effective with regard to tremor suppression than thalamotomy with a lower overall rate of adverse events; however, one mortality was reported in the stimulation group related to intracerebral hematoma. ${ }^{9}$ The morbidity associated with historical surgical techniques has led to exploration of additional procedural tools.

Since 2010, there has been new attention given to the use of FUS for the treatment of ET with the publication of multiple pilot trials. Lipsman et al. published a small, open-label study which included outcomes of unilateral thalamotomy in four patients, targeting the Vim. Patients were followed for 3 months with outcomes measured by scores on the Clinical Rating scale for Tremor (CRST). An 81.3\% reduction in tremor was reported at 3 months. Participants additionally reported a $45.5 \%$ improvement in fine motor tasks (writing, drawing), and $51.1 \%$ reduction in subjective functional disability at 3 months. The adverse effects included persistent finger paresthesias in one patient at 3 months, and a deep venous thromboembolism 1 week after the procedure in another patient. ${ }^{15}$

Chang et al. published a feasibility trial, in which 11 participants were recruited to undergo unilateral focused-ultrasound thalamotomy. Of the 11 participants, only eight reached therapeutic temperature. These patients were followed for 6 months at which time they reported improvement in parts $A, B$, and $C$ of the CRST. Parts A and B involve clinician/investigator rating of tremor severity with a patient writing, drawing spirals, pouring water, and other physical exam tasks. Part C of the CRST relates to patient-reported disability due to tremor. Despite the participants' clinical improvement, MRI lesions disappeared after 1 month. There were minimal intra-procedural side effects reported. One patient experienced mild balance problems following the procedure, but this resolved with 1 month of steroids. ${ }^{16}$

Elias et al. published a larger, open-label study, in which 15 patients were recruited for unilateral FUS thalamotomy targeting the Vim. Followup extended to 12 months with outcomes measured on CRST. Patients experienced a relative reduction in contralateral tremor of $75 \%$ from baseline, at 12 months. There was also significant improvement in disability, improved quality of life, and improved physical function $(p=0.001)$. Immediate adverse effects included transient contralateral paresthesias and unsteadiness. One patient developed persistent dysesthesias of a single finger. ${ }^{17}$

Building on the results of their pilot study, Elias et al. went on to publish the pivotal trial18 which led to US Food and Drug Administration (FDA) approval of MRgFUS for treatment of ET in July 2016. This multicenter, prospective,
Figure 1: Aspects of magnetic resonance imaging-guided focused ultrasound ${ }^{18}$
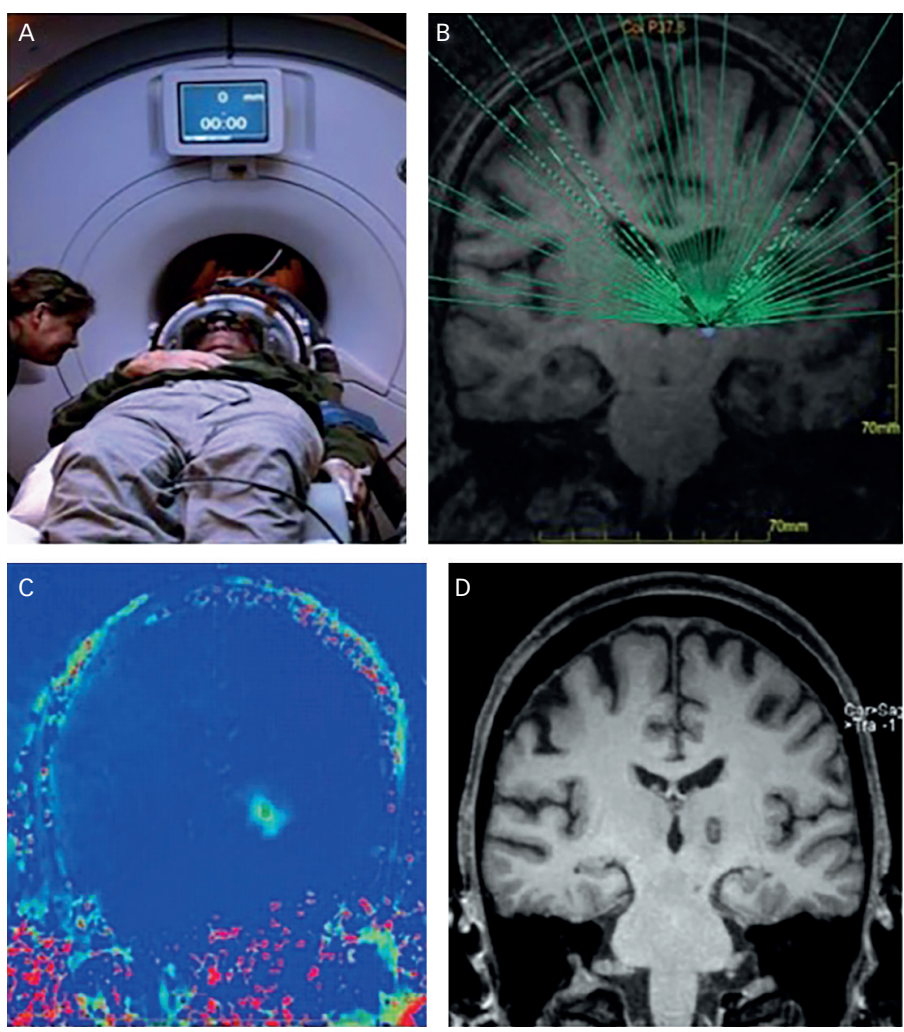

Included with permission of Dr Elias and reproduced from the New England Journal of Medicine. ${ }^{18}$ Panels demonstrate A: patient positioning, B: 1,024 ultrasonic transducers arranged in hemispheric orientation, $\mathrm{C}$ : thermometry guidance, and D: magnetic resonance imaging lesion.

randomized trial confirmed the results of prior pilot studies. Elias et al. recruited 76 patients with moderate to severe ET. Study participants were randomized 3:1 to receive ultrasound-guided thalamotomy versus sham procedure. Patients and assessors were blinded to treatment group. Hand tremor severity (based on the CRST) improved by $47 \%$ at 3 months in the treatment group, compared to $0.1 \%$ in the sham group. This effect was sustained at 12 months with a $40 \%$ improvement from baseline of tremor severity in the treatment group. In addition to tremor improvement, the individuals who underwent FUS thalamotomy had improved overall disability scores and improved quality of life (based on the Quality of Life in Essential Tremor Questionnaire [QUEST]), with the most significant improvement noted in the psychosocial domain. Adverse effects during this trial included gait disturbance in 36\% and sensory disturbance in 38\%; these symptoms did not persist in the majority of patients at 12 months ( $9 \%$ and $14 \%$, respectively). ${ }^{18}$ This study was the first to provide robust data demonstrating the effect of MRgFUS thalamotomy, with sustained improvements noted in tremor severity, disability, and quality of life.

Though FUS has proven to be safe and effective for unilateral thalamotomy in patients with ET, further work will be required to determine durability of benefit. With this consideration, it will also be important to see if tremor re-emerges and if retreatment with MRgFUS thalamotomy has value. While lesioning procedures have typically been used for unilateral treatments only, understanding the safety and therapeutic effects of bilateral lesioning may be explored in the future. As such, comparing concurrent versus 
staged procedures will be important. A study published in 2016 by Gallay et al. demonstrated, in a small number of patients with ET, that bilateral lesioning of the cerebellothalamic tract could be effectively performed in staged procedures, though with increased gait instability reported as an adverse effect. ${ }^{19}$ Additional questions include what, if any, long-term cognitive effects of FUS thalamotomy in ET may have, particularly with bilateral procedures.

\section{Parkinson's disease}

Surgical treatment for PD has been explored since the early 20th century. ${ }^{20,21}$ Due to high morbidity and mortality of initial open procedures, the technique of closed stereotactic functional neurosurgery was developed starting in the 1940s. With the advent of levodopa in the late 1960s, surgical treatments for PD fell out of favor and were not re-examined rigorously until the 1980s as a way to target the motor fluctuations associated with chronic levodopa use.22 DBS was developed during that time and approved by the FDA for treatment of PD in 1997. Over the course of several decades, the target for DBS in PD has shifted to preferentially include the globus pallidus interna (GPi) and subthalamic nucleus (STN). Because of the ability to modulate its effects, DBS has become the favored surgical option for treatment of PD. Thalamotomy and pallidotomy are now usually reserved for select individuals, including those with surgical contraindications, those desiring palliative treatments, and those with strong preference away from DBS. The successful use of FUS in the ET population, however, has led to further exploration of this technique for PD. Currently, data on the safety and utility of FUS lesioning in PD has been limited but is being vigorously explored.

\section{Thalamus}

The Vim of the thalamus has been a target for lesioning procedures for treatment of tremor in PD for many years. MRgFUS thalamotomy in PD has recently been explored.

Magara et al. enrolled 13 patients with treatment-resistant PD. The majority of participants suffered from tremulo-akinetic disease phenotype. The pallidothalamic tract was targeted with the first group of patients receiving a single application of ultrasound energy, and the second group receiving multiple applications of energy (four to five in total). Follow-up examinations were performed at 3 months. This study demonstrated that repeated application of energy allowed for more sustained effects with a 60.9\% mean total Unified Parkinson's Disease Rating Scale (UPDRS) score reduction noted in the second group, versus $7.6 \%$ reduction in the first group at 3 months. ${ }^{23}$ There were no procedure-related side effects reported in either group. Based on the Mini Mental Status Test and the Hospital Anxiety and Depression Scores, there was no change in mood or cognition pre and post procedure for either group.

Schlesinger et al. studied seven patients with moderate to severe Parkinson tremor, performing unilateral FUS Vim thalamotomy to the side with most severe tremor. ${ }^{24}$ Patients were followed for 3-12 months. Results showed a significant improvement at 1 week on the mean UPDRS (from $37.4 \pm 12.2$ points to $18.8 \pm 11.1, p=0,007$ ), UPDRS Item 20 mean score (from $2.7 \pm 1.1$ to $0.0 \pm 0.0 . p<0.001$ ) and UPDRS Item 21 mean score (from $3.0 \pm 1.0$ to $0.0 \pm 0.0, p<0.001)$. Quality of life as measured by the Parkinson's Disease Questionnaire (PDQ39) also improved significantly at 1 week $(42.3 \pm 16.4$ to $21.6 \pm 10.8, p=0.008)$. These improvements persisted at 3 months. Side effects included hypogeusia, subjective gait instability and difficulty tandem walking, the latter two of which resolved. The Mini Mental Status Exam scores were stable before and after the procedure. ${ }^{24}$

A randomized, sham-controlled trial by Bond et al. was recently completed with enrollment of 27 subjects (in submission). The primary outcome measured tremor improvement between the procedure and sham groups at 3 months based on the CRST. Preliminary results show a 50\% improvement in hand tremor at 3 months in patients undergoing FUS despite a strong placebo effect, with $22 \%$ improvement reported in the sham group. Outcome at 1 year showed a $40.6 \%$ improvement in tremor scores in the treatment group along with $32 \%$ reduction in UPDRS motor scores $\left(p=0.088\right.$ and 0.033 , respectively). ${ }^{25}$ subjects undergoing sham treatment also showed improvement in both measures. While there was a significant difference between active and sham groups in tremor reduction measured by CRST, the difference in UPDRS part III was not significant. Cognitive measures were not different between the groups at 3 months.

Prior studies in the PD population have suggested that cognition may be affected following thalamotomy, particularly when performed in the left hemisphere. ${ }^{26}$ This risk for this effect following MRgFUS thalamotomy is currently unknown but will be important to study in the future.

\section{Subthalamic nucleus}

The anti-tremor effects of either ablation or stimulation of the STN have historically made it an attractive target for functional neurosurgery in PD. Prior reviews have suggested that unilateral subthalamotomy not only improves the cardinal features of PD in the on and off medication states, but additionally allows for a substantial decrease in the daily requirement of levodopa, thereby often leading to decreased dyskinesia. Side effects from unilateral STN ablation can include hemiballismus/hemichorea and postural instability, though these are typically transient. ${ }^{27}$ The effects of FUS on the STN are unclear at this time but multiple trials are currently under way to explore this (ClinicalTrials.gov Identifiers: NCT02003248 and NCT02252380). As of yet, there are no published preliminary data from these studies available.

\section{Globus pallidus}

The GPi has been a favored target for lesioning and DBS particularly due its effects on suppressing dyskinesia in $\mathrm{PD}$, though there is also well characterized benefit in bradykinesia and rigidity. A case report from $\mathrm{Na}$ et al. has demonstrated safety and efficacy of FUS pallidotomy in a single patient with PD with sustained effects 6 months later. This patient experienced an $80 \%$ reduction in the UPDRS Part I score, a 51\% reduction in the Part II score, and a 60\% and 54\% reduction in the Part III score "on" and "off" medication, respectively, by 6 months. No adverse effects of the procedure were documented. ${ }^{28}$ A multicenter feasibility trial is currently underway exploring unilateral FUS-guided lesioning of the globus pallidum for medically-refractory dyskinesias in advanced PD.

\section{Use of focused ultrasound in other movement disorders}

There is limited data, at present, for the use of FUS in less common movement disorders. The safety and efficacy of MRgFUS in treatment of ET and PD would suggest that this technique could be successfully applied to other movement disorders such as chorea or dystonia. The use of DBS in dystonia is well established with the target typically the GPi, and other less studied targets including the STN or the thalamus. In 2017, researchers from Tokyo published a case report of an individual with focal hand dystonia who 
was successfully treated with MRgFUS to the ventrooralis nucleus of the thalamus. ${ }^{29}$ One case report demonstrated efficacy of MRgFUS in tremor associated with Fragile $X$ tremor ataxia syndrome (FXTAS). ${ }^{30}$ There are no trials published evaluating MRgFUS in the treatment of chorea.

\section{The future of focused ultrasound Low-intensity focused ultrasound}

While high-intensity FUS lesioning has received the most recent attention, research into low-intensity FUS (LIFUS) is gaining momentum in the field of neuromodulation. Though multiple modalities for neural stimulation are currently available, they either require an invasive surgery (in the case of DBS and vagal nerve stimulation) or lack target specificity (in the case of transcranial magnetic stimulation and cranial electrotherapy stimulation). LIFUS could provide a non-invasive precise targeting technique for neuromodulation. While the exact mechanism of LIFUS is unknown, prior studies have suggested that lower power sonications can cause either inhibitory or excitatory effects which are reversible. ${ }^{31}$ The threshold for thermal ablation is not met with lower intensity sonications. Tufail et al. observed clinical motor contraction following stimulation of the motor cortex of mice, ${ }^{32}$ while others have described reversible suppression of visual-evoked potentials using LIFUS. ${ }^{32}$ The potential uses of LIFUS not only in neuromodulation but also brain mapping suggest that this is a novel and emerging technology; however, the efficacy and duration of effect of this technique is currently unknown.

\section{Use of focused ultrasound in other diseases}

FUS is not only under review for use in multiple movement disorders, but is being applied as a treatment for many other neurologic and non-neurologic diseases. These include, but are not limited to, treatment of neuropathic pain, ${ }^{3}$ tumor ablation, ${ }^{34}$ and sonothrombolysis for stroke therapy. ${ }^{35}$ MRgFUS is also being studied as a means for drug delivery across the blood-brain barrier, which may have implications for treating brain tumors, demyelinating disorders, neuroinflammatory conditions, and other disorders where targeted therapy may be indicated..$^{36,37}$

\section{Summary}

Though various surgical techniques have been adopted as treatment methods for various movement disorders, the development of FUS for lesioning procedures adds a new treatment paradigm. MRI guidance intraprocedurally, phased-array transducers, and real-time clinical feedback allow for precise lesioning using FUS techniques. MRgFUS has been shown to be effective and safe. The most robust evidence for use of this technique comes from studies targeting patients with ET with reproducible effects following FUS thalamotomy including a sustained decreased tremor, decreased disability and improved quality of life. These studies led to approval of MRgFUS for the treatment of ET in 2016. Studies exploring the efficacy and safety of MRgFUS in Parkinson's patients for treatment of tremor or dyskinesias are currently under way with promising preliminary data; however, the ideal ultrasound target in this disease has been yet to be defined. There is a paucity of data regarding use of MRgFUS in rarer movement disorders though individual case reports have also been published demonstrating efficacy in FXTAS tremor. ${ }^{38}$ Larger studies will be required to define, not only the role of MRgFUS for these rarer diseases and the long-term effects of unilateral versus bilateral lesioning, but also the role of this treatment vis-à-vis currently utilized and effective therapies such as DBS. $]$
1. Fry WJ, Mosberg WH Jr, Barnard JW, Fry FJ, Production of focal destructive lesions in the central nervous system with ultrasound, J Neurosurg, 1954;11:471-8.

2. Hynynen K, Vykhodtseva NI, Chung AH, et al., Thermal effects of focused ultrasound on the brain: determination with MR imaging, Radiology, 1997; 204:247-53.

3. Jeanmonod D, Werner B, Morel A, et al., Transcranial magnetic resonance imaging-guided focused ultrasound: noninvasive central lateral thalamotomy for chronic neuropathic pain, Neurosurg Focus, 2012;32:E1.

4. Louis ED, Ferreira JJ, How common is the most common adult movement disorder? Update on the worldwide prevalence of essential tremor, Mov Disord, 2010;25:534-41.

5. Louis ED, Clinical practice. Essential tremor, N Engl J Med, 2001;345:887-91.

6. Zesiewicz TA, Elble RJ, Louis ED, et al., Evidence-based guideline update: treatment of essential tremor. Report of the quality standards subcommittee of the American Academy of Neurology, Neurology, 2011;77:1752-5

7. Benabid AL, Pollak P, Gao D, et al. Chronic electrical stimulation of the ventralis intermedius nucleus of the thalamus as a treatment of movement disorders, J Neurosurg, 1996;84:203-14.

8. Zesiewicz TA, Elble R, Louis ED, et al., Practice parameter: therapies for essential tremor. Report of the quality standards subcommittee of the American Academy of Neurology, Neurology, 2005;64:2008-20.

9. Schuurman PR, Bosch DA, Bossuyt PM, et al., A comparison of continuous thalamic stimulation and thalamotomy for suppression of severe tremor, N Eng/ J Med, 2000;342:461-8.

10. Ohye C, Higuchi Y, Shibazaki T, et al., Gamma knife thalamotomy for Parkinson disease and essential tremor: a prospective multicenter study, Neurosurgery, 2012;70:526-35; discussion 535-6.

11. Kondziolka D, Ong JG, Lee JY, et al., Gamma knife thalamotomy for essential tremor, J Neurosurg, 2008;108:111-7.

12. Witjas T, Carron R, Krack P, et al., A prospective single-blind study of gamma knife thalamotomy for tremor, Neurology, 2015;85:1562-8.

13. Young RF, Li F, Vermeulen S, Meier R, Gamma knife thalamotomy for treatment of essential tremor: long-term results, I Neurosurg 2010;112:1311-7
14. Koller W, Pahwa R, Busenbark K, et al., High-frequency unilateral thalamic stimulation in the treatment of essential and Parkinsonian tremor, Ann Neurol, 1997;42:292-9.

15. Lipsman N, Schwartz ML, Huang Y, et al., MR-guided focused ultrasound thalamotomy for essential tremor: a proof-of-concept study, Lancet Neurol, 2013;12:462-8.

16. Chang WS, Jung HH, Kweon EJ, et al., Unilateral magnetic resonance guided focused ultrasound thalamotomy for essentia tremor: practices and clinicoradiological outcomes, J Neurol Neurosurg Psychiatry, 2015;86:257-64.

17. Elias WJ, HusS D, Voss T, et al., A pilot study of focused ultrasound thalamotomy for essential tremor, N Engl J Med, 2013;369:640-8.

18. Elias WJ, Lipsman N, Ondo WG, et al., A randomized trial of focused ultrasound thalamotomy for essential tremor, NEng/ J Med, 2016;375:730-9.

19. Gallay MN, Moser D, Rossi F, et al., Incisionless transcranial MRguided focused ultrasound in essential tremor: cerebellothalamic tractotomy, J Ther Ultrasound, 2016;4:5. DOI: 10.1186/s40349016-0049-8.

20. Speelman JD, Bosch DA, Resurgence of functional neurosurgery for Parkinson's disease: a historical perspective, Mov Disord, 1998;13:582-8.

21. Meyers R, Fry WJ, Fry FJ, et al., Early experiences with ultrasonic irradiation of the pallidofugal and nigral complexes in hyperkinetic and hypertonic disorders, J Neurosurg, 1959;16:32-54.

22. Krack P, Martinez-Fernandez R, Del Alamo M, Obeso JA, Current applications and limitations of surgical treatments for movement disorders, Mov Disord, 2017;32:36-52

23. Magara A, Buhler R, Moser D, et al., First experience with MR-guided focused ultrasound in the treatment of Parkinson's disease, I Ther Ultrasound, 2014;2:11. doi: 10.1186/2050-57362-11

24. Schlesinger I, Eran A, Sinai A, et al., MRI guided focused ultrasound thalamotomy for moderate-to-severe tremor in Parkinson's disease, Parkinsons Dis, 2015;2015:219149.

25. Bond AE, Dallapiazza R, Huss $D$, et al., $132 \mathrm{~A}$ randomized, sham-controlled trial of transcranial magnetic resonance-guided focused ultrasound thalamotomy trial for the treatment of tremordominant, idiopathic Parkinson disease, Neurosurgery, 2016;63 Suppl 1:154.

26. Nijhawan SR, Banks SJ, Aziz TZ, et al., Changes in cognition and health-related quality of life with unilateral thalamotomy for Parkinsonian tremor, I Clin Neurosci, 2009;16:44-50.

27. Jourdain VA, Schechtmann G, Di Paolo T, Subthalamotomy in the treatment of Parkinson's disease: clinical aspects and mechanisms of action, J Neurosurg, 2014;120:140-51.

28. Na YC, Chang WS, Jung HH, et al., Unilateral magnetic resonanceguided focused ultrasound pallidotomy for Parkinson disease, Neurology, 2015:85:549-51.

29. Horisawa S, Tamura N, Hayashi M, et al., Gamma knife ventro-oral thalamotomy for musician's dystonia, Mov Disord, 2017;32:89-90.

30. Cerquera C, Rumia J, Herrera JM, et al., A single case report of MR-guided focused ultrasound thalamotomy for tremor in fragile X-associated tremor/ataxia, Parkinsonism Relat Disord, 2016;28:159-60

31. Bystritsky A, Korb AS, Douglas PK, et al., A review of low-intensity focused ultrasound pulsation, Brain Stimul, 2011;4:125-36.

32. Tufail Y, Matyushov A, Baldwin $N$, et al., Transcranial pulsed ultrasound stimulates intact brain circuits, Neuron, 2010;66:681-94.

33. Kim H, Park MY, Lee SD, et al., Suppression of EEG visual-evoked potentials in rats through neuromodulatory focused ultrasound NeuroReport, 2015;26:211-5.

34. Jolesz FA, Hynynen K, McDannold N, et al., Noninvasive thermal ablation of hepatocellular carcinoma by using magnetic resonance imaging-guided focused ultrasound, Gastroenterology ، 2004;127(5 Suppl 1):S242-7.

35. Damianou C, Hadjisavvas V, Ioannides K, In vitro and in vivo evaluation of a magnetic resonance imaging-guided focused ultrasound system for dissolving clots in combination with thrombolytic drugs, I Stroke Cerebrovasc Dis, 2014;23:1956-64

36. MCDannold N, Arvanitis CD, Vykhodtseva N, Livingstone MS, Temporary disruption of the blood-brain barrier by use of ultrasound and microbubbles: safety and efficacy evaluation in rhesus macaques, Cancer Res, 2012;72:3652-63.

37. O'Reilly MA, Waspe AC, Chopra R, Hynynen K, MRI-guided disruption of the blood-brain barrier using transcranial focused ultrasound in a rat model, J Vis Exp, 2012. pii: 3555.

38. Fasano A, Sammartino F, Llinas M, Lozano AM. MRl-guided focused ultrasound thalamotomy in fragile $X$-associated tremor/ ataxia syndrome, Neurology, 2016;87:736-8. 